\title{
Evaluation of Quality of Websites Related to Sexaholics Anonymous: Improvement of Online Patient Education in High Risk Sexual
} Behaviors

\author{
Parisa Enayati ${ }^{1}$, Pooria Sobhanian Kafshgarkolaee ${ }^{2}$, Ali Asghar Nadi Ghara ${ }^{3}$ and Raheleh Rafaiee ${ }^{4,{ }^{*}}$ \\ ${ }^{1}$ Psychiatry and Behavioral Sciences, Addiction Research Institute, Mazandaran University of Medical Sciences, Sari, IR Iran \\ ${ }^{2}$ Student Research Committee, School of Medicine, Mazandaran University of Medical Sciences, Sari, IR Iran \\ ${ }^{3}$ Health Sciences Research Center, Addiction Research Institute, Mazandaran University of Medical Sciences, Sari, IR Iran \\ ${ }^{4}$ Department of Neuroscience, School of Advanced Technologies in Medicine, Mazandaran University of Medical Sciences, Sari, IR Iran \\ "Corresponding author: Department of Neuroscience, School of Advanced Technologies in Medicine, Mazandaran University of Medical Sciences, Sari, IR Iran. Email: \\ rahele_rafaie@yahoo.com
}

Received 2019 April 18; Revised 2020 January 13; Accepted 2020 February 18.

\begin{abstract}
Background: The internet advising system is growing as the essential means of health. Regarding the importance of addictive disorders as well as the requirement for websites to provide up-to-date information about such disorders and inform patients how to prevent and treat them, the structure and content of these websites are extremely crucial.

Objectives: This research aimed to evaluate websites presenting information on treatment for sex addiction in Sexaholics Anonymous (SA) to users and also to compare the quality of related Persian and English websites.

Patients and Methods: This cross-sectional study reviewed six websites addressing SA found through the use of the Google and Yahoo search engines by searching four key terms (behavioral addiction, sex addiction, sex, treatment of sex addiction). Two Persian and four English websites related to SA were found. Each website was assessed using the Website Quality Evaluation Tool by 42 research assistants based on indicators including content, functionality, being up-to-date, links, graphics, authority, coverage and style.

Results: Overall, the quality of websites related to SA was "trustable". There was no significant difference between the total scores of the quality of the SA-related websites. Moreover, a significant difference was found between the English and Persian websites in terms of being up-to-date sub-criterion $(\mathrm{P}=0.007)$.

Conclusions: The websites related to SA met the user's needs. The scores of the Persian websites were lower than those of the English ones. Treatment programs for sex addiction within the Iran health system remain limited. This study suggests that we should use technology in national healthcare services, especially in the area of web and databases.
\end{abstract}

Keywords: Sex, Sexual Behavior, Sex Education, Internet, Evaluation Study, Behavior, Addictive

\section{Background}

The Internet has been found as one of the important sources of health information. Online information search is usually the beginning effort to seek diagnostic criteria and treatment of diseases (1). The internet advising system will grow as the essential means of health in the future (2). It presents an appropriate approach for improvement of health and efficient distribution of knowledge about taboo subjects, particularly in traditional cultures (3).

Sex addiction is unlike any other taboo issues and is a complicated one. It has been considered as either a guilt or a disease, depending on the religious or political viewpoint at the time (4). This uncontrollable, extreme, and problematic sexual behavior has been explained by applying sev- eral various descriptions including hypersexuality, sexual compulsion, sexual impulsivity, sexual addiction, and sexual dependency (5).

The prevalence of sex addiction is determined at around $3 \%$ to $6 \%$ of the general community, with a significantly higher proportion of males than females. High sex addiction rates are reported in special groups such as HIV affected patients and sexual offenders. Sex addiction may be more prevalent in homosexual and bisexual men (6). The private nature of sex and the stigma associated with these behaviors potentially cause these behaviors to remain hidden due to embarrassment or shame. Behaviors such as unrestricted sex living, masturbation, and sexual desire are forbidden, and according to spiritual and social 
perspectives, they are not moral and are judged as sinful (7).

There are few studies on sex addiction in Iran because the taboo nature of the subject (8). The prevalence rate of sex addiction and its outcomes in Iran and elsewhere is currently unknown (9). There are no FDA-approved medications for sex addiction (10). Various social barriers and limitations exist in seeking treatment for sex addiction (11). Thus, affected individuals may be further prone to explore for treating sex addiction through an internet search (12). Sex act, even if it is not complicated, is related to shame in the culture of Muslims, and the sex addicted might be less inclined to inquire therapy for sex addiction in religious cultures (13).

Anonymity associated with Internet facilitates can increase the online user's feeling of comfort (14). Sex is the most frequently searched subject on the Internet (15). Despite the potential addictiveness of the Internet (16) and its relationship with online sexual behaviors (17), the Internet can be used for treatment related purposes toward sexual problems (18). These include the use of the Internet for seeking out sexually related websites for educational use (19), seeking out sex therapists (20), and seeking recovery in programs of anonymous self-help groups (21). Self-help groups, utilizing the Alcoholics Anonymous model (a successful support group for Alcoholism) to sexual behavior, have developed in many countries in recent years (22). sexaholics anonymous (SA) is a non-governmental organization (NGO) consisting of people whose main problem is sex (23). The members of this association share their experiences in regular open meetings and keep their recovery through 12-Steps and 12-Traditions (24).

In order to increase the hidden growing of sexual crime and help-seeking sex addicted individuals in Iran (25), it is necessary to identify the facilities that may impact treatment access amongst them. Treatment programs for sex addiction within the Iran health system remain limited (9); however, SA helps patients affected with sex addiction in Iran. Among the Muslim countries in the Middle East, Iran is the only country where SA is activated and its regular meetings are held. The 15th anniversary of SA's activities was celebrated in Iran, in 2018 (26). Despite the importance of the activation of this NGO in Iran, it is still not well known. Therefore, introduction of websites related to SA in Iran and improvement of their quality are important.

With regard to the importance of addictive disorders as well as the requirement for websites to provide upto-date information about such disorders and inform patients how to prevent and treat them, the structure and content of these websites are extremely critical (27).

\section{Objectives}

The purpose of this paper was to evaluate the quality of available websites related to SA treatment and consultation about it to help identify sex addicted patients in Iran. Moreover, SA websites in Iran and other countries were compared.

\section{Patients and Methods}

The present descriptive cross-sectional study was designed and carried out in January, 2019. For the purpose of data collection, 42 research assistants selected from Iranian volunteer medical students in student research committees, chose websites through direct observation of each website and careful review of them. Our research assistants were medical students because they are capable of easily recognizing the content of health websites. All the respondents were routine internet users and familiar with the computer, the Internet and English language. First, for recognizing SA-related websites, Persian equivalences of four search terms (behavioral addiction, sex addiction, sex, treatment of sex addiction) were searched in Google and Yahoo because of their popularity among Iranian people. Finally, we found two Persian and four English websites related to SA.

The Website Quality Evaluation Tool (WQET) was designed to determine the overall web quality. The WQET evaluates eight criteria (content, functionality, being upto-date, links, graphics, authority, coverage, and style) with 36 questions. In WQET, each question is scored in a scale of 1 to 7. The total score of quality was obtained from eight different criteria with scores ranging from 1 to 7 (1 - 2 weak, 3 - 4 moderate, 5 - 6 good, and 7 excellent) for the different existing sub-criteria. Three criteria including content, performance, and reliability were measured by doubling their privileges. The reason that these three criteria were doubled was that in this case, the content would refer to the content of thought, novelty and clarity of the content. Functionality means ease of use and search, and reliability refers to the credit of the creator or the backup organization.

Measuring these factors indicates the significance of the subject and the website quality experts' attention to quality. The maximum normalized scale was 82 for each website; in WQET, the lowest score is less than 50.

For the normalizing score, the following formula was used:

Score of website,

$A=\frac{(X \times 82)}{Y}$

Where, $\mathrm{X}$ is the total score for each website and $\mathrm{Y}$ is the top score of one website. In addition, the normalized score 
was coded according to McInerney and Bird (2005). If the overall quality score of a website is between $71-82$, the website is great and trustworthy. If the overall quality score of a website is between $64-70$, it is acceptable and can be remembered. A website with a quality score between 57-63 is good but should be used cautiously. If a website has a quality score between 50 - 56, it may be useful in some cases, but the information it provides cannot be trusted without research and the website is only relatively good. Finally, a website with the score below 50 is inappropriate and its information is not sufficient and valid. The reliability of the current measurement instruments, Cronbach's alpha was 95\%, shows very high reliability (28). The data were analyzed using SPSS V.18 and Excel software. Comparisons between the variables were computed using one-way ANOVA and Duncan's multiple range test (post hoc). The level of significance was set at 0.05 .

\section{Results}

The coding results of the six examined websites showed that "Sa.org" belonging to the SA International Central Office ranked first based on the WQET instrument. The coding results of the other websites in WQET were as follows: "Saa.recovery.org" was 81.57, "Saa.recovery.org.uk" was 80.93, "Saoz.net" was 74.75, "Sa-Iran.org" was 71.60 and "Saesfahan.ir" was 68.66. The first five websites were classified as excellent websites based on their scores, while the last website was classified as a very good website based on its score. Generally, the coding rates of the Persian websites were lower than those of their English counterparts. Figure 1 shows the ranking of the SA-related websites based on the normalized scores.

Since the data followed a normal distribution (based on the Shapiro-Wilks test), we used one-way ANOVA. There was no significant difference between the total quality scores of these websites (Table 1 ).

Table 2 shows detailed results of the six examined websites based on the WQET instrument. A significant difference was found between the websites in terms of being upto-date. However, no other statistically significant differences were found between the other sub-criteria.

Table 3 shows comparison of the mean scores of being up-to-date from Table 2 using Duncan's multiple range test (posthoc).

\section{Discussion}

This is the first study to assess whether websites related to SA meet users' information needs. The results showed that the quality of SA information on the websites was generally reliable based on the WQET instrument. The top websites were ranked as follows: www.sa.org, www.saa.recovery.org, www.saa.recovery.org.uk, www.saoz.net, www.sa-Iran.org, and www.saesfahan.ir. Internet users often do not go beyond the first page of their search results; therefore, they may overlook websites that provide high-quality information. In Google ranking of SA-related websites, the first website is www.sa.org whereas other high-quality websites are not indexed properly in Google and may be missed.

Based on the present findings, the quality of Persian SA-related websites is lower than that of English SA-related ones. Our results are in agreement with previous studies, as they showed that the mean total quality score of Persian websites on addiction was 64.57 according to the WQET instrument; therefore, the websites were ranked as "very good" on average. Lack of online services, such as chat rooms, was a restriction that reduced users' satisfaction with Persian websites on addiction. In contrast, the scores of Persian addiction websites were low and moderate based on the Silberg and DISCERN instruments, respectively (29).

Several studies have highlighted the quality of webbased information about addiction. Evaluation of the quality of web-based information on cocaine (29), cannabis, and addiction (30) showed that the overall quality of websites for patients was weak, based on the "Health on the Net" (HON) and DISCERN instruments. Moreover, the quality evaluation of French websites on alcohol dependency revealed that the quality of these websites was almost poor, particularly regarding feasible therapies (31). The cause of discrepancy between our results and some previous research is the use of different tools. In fact, in recent years, many tools have become available for reviewing websites providing health information (32).

It is generally agreed that content quality is an important dimension of websites, which deals with characteristics of available information on websites. Comparison of the English websites with the Persian ones showed that the English websites obtained higher scores regarding the content sub-criterion. Many studies on consumer health information of websites showed significant drawbacks in their content, such as incomplete and inaccurate online health information. In this regard, Eysenbach et al. conducted a systematic review of characteristics of 79 studies evaluating the quality of health information on websites. Based on their findings, 70\% of previous studies concluded that the quality of information on the Internet was low, $21 \%$ reported the neutral quality of information, and 9\% reported more positive findings (27). The existing literature indicates that the content of online health information is hard to understand by the majority of people (33). We recruited individuals with professional medical background to evaluate the quality of SA-related websites and adjust 


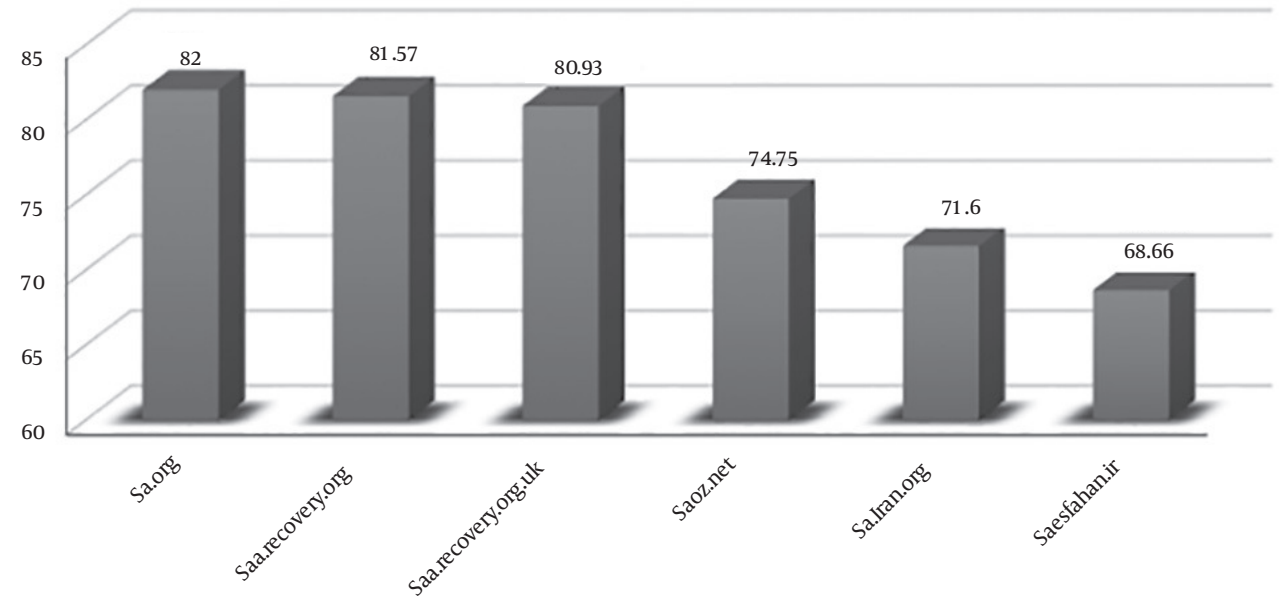

Figure 1. Normalized scores of SA-related websites based on the WQET instrument

Table 1. Total Scores of the Quality of the SA-Related Websites

\begin{tabular}{lllll}
\hline Websites & N & Mean & SD & P Value \\
\hline Sa.org & 7 & 263.57 & 48.36 & 90.13 \\
\hline Saa.recovery.org & 7 & 262.20 & 46.44 & 0.855 \\
\hline Saa.recovery.org.uk & 7 & 260.14 & 39.92 & 30.88 \\
\hline saoz.net & 7 & 240.29 & $0.5210^{\mathrm{b}}$ & 50.43 \\
\hline Sa-iran.org & 7 & 230.14 & 220.71 & \\
\hline Saesfahan.ir & 7 & . & \\
\hline
\end{tabular}

${ }^{\mathrm{a}}$ The total scores and ANOVA results of the six examined websites based on the WQET scale.

${ }^{\mathrm{b}}$ Not Significant.

Table 2. Sub-Criteria Scores of the WQET Scale of the SA-Related Websites ${ }^{\mathrm{a}}$

\begin{tabular}{|c|c|c|c|c|c|c|c|c|}
\hline Criteria & Saa.recovery.org.uk ${ }^{b}$ & Saoz.net ${ }^{c}$ & Sa.org ${ }^{d}$ & Saa.recovery.org ${ }^{\mathbf{e}}$ & Sa-iran.org ${ }^{f}$ & Saesfahan.ir ${ }^{\mathrm{f}}$ & $\mathbf{F}$ & P Value \\
\hline Content & $71.14 \pm 16.44$ & $73.14 \pm 16.40$ & $72 \pm 15.23$ & $70.4 \pm 19.15$ & $63.14 \pm 10.31$ & $62.86 \pm 10.20$ & 0.594 & 0.705 \\
\hline Functionality & $63.14 \pm 13.20$ & $54.86 \pm 6.48$ & $62.29 \pm 17.90$ & $59.6 \pm 19.41$ & $53.71 \pm 19.90$ & $51.14 \pm 21.53$ & 0.505 & 0.771 \\
\hline Being up-to-date & $15.36 \pm 4.77$ & $15 \pm 3.95$ & $16.14 \pm 3.62$ & $11.60 \pm 7.60$ & $8.43 \pm 4.31$ & $8.71 \pm 4.46$ & 3.829 & $0.007^{g}$ \\
\hline Links & $15.14 \pm 3.33$ & $12.29 \pm 4.78$ & $16.14 \pm 3.62$ & $15.2 \pm 3.76$ & $12.71 \pm 2.43$ & $12.57 \pm 2.76$ & 1.911 & 0.118 \\
\hline Graphics & $23.86 \pm 9.37$ & $24 \pm 8.90$ & $31 \pm 7.11$ & $34.40 \pm 28.89$ & $28.86 \pm 5.9$ & $25.71 \pm 5.88$ & 0.721 & 0.612 \\
\hline Authority & $38 \pm 13.9$ & $28.29 \pm 6.77$ & $30.71 \pm 8.77$ & $38.80 \pm 12.29$ & $28.29 \pm 8.59$ & $25.43 \pm 8.22$ & 1.961 & 0.110 \\
\hline Coverage & $15.29 \pm 3.03$ & $14.14 \pm 5.36$ & $16 \pm 3.91$ & $13.40 \pm 6.33$ & $15.14 \pm 4.77$ & $14.14 \pm 4.41$ & 0.262 & 0.931 \\
\hline Style & $17.71 \pm 1.79$ & $18.57 \pm 2.57$ & $18.86 \pm 5.17$ & $18.80 \pm 6.30$ & $19.86 \pm 3.02$ & $20.14 \pm 3.07$ & 0.381 & 0.858 \\
\hline
\end{tabular}

${ }^{\mathrm{a}}$ Mean \pm SD scores.

${ }^{\mathrm{b}}$ Location of Website: United Kingdom.

${ }^{c}$ Location of Website: Australia.

${ }^{\mathrm{d}}$ Location of Website: United States and Canada.

${ }^{\mathrm{e}}$ Location of Website: United States.

${ }^{\mathrm{f}}$ Location of Website: Iran.

${ }^{\mathrm{g}}$ Significant differences were detected between the websites in terms of being up-to-date.

their content with scientific data.

Our results are similar to those of previous studies, which showed major problems in updating the website information about treatments $(29,34)$. According to the present results, a significant difference was found between the English and Persian websites in terms of being updateto-date sub-criteria. The need for recording the date of information update is one of the most important criteria, which was neglected in the Persian SA-related websites. Generally, updating is an important facet of health infor- 
Table 3. Comparison of the Mean Scores of Being Up-to-Date Using Duncan's Multiple Range Test ${ }^{\mathrm{a}}$

\begin{tabular}{lcc}
\hline & \multicolumn{2}{c}{ Subset for Alpha $=0.05$} \\
\cline { 2 - 3 } Websites & $\mathbf{1}$ & $\mathbf{2}$ \\
\hline Sa-iran.org & 8.43 & \\
Saesfahan.ir & 8.71 & \\
\hline Saa.recovery.org & 11.60 & 11.60 \\
\hline saoz.net & & 15.00 \\
\hline Saa.recovery.org.uk & & 15.86 \\
\hline Sa.org & & 16.14 \\
\hline Sig. & 0.264 & 0.123 \\
\hline
\end{tabular}

${ }^{\mathrm{a}}$ Means for groups in homogeneous subsets are displayed. The harmonic mean of the group sizes is used.

mation, as medical opinions and research findings evolve over time with the introduction of new treatments and information. It is important to keep patients up-to-date, especially when their condition is not well understood. Furthermore, people may assume that information available on websites is up-to-date. However, many websites do not satisfy the updating criterion. Persian SA-related websites need to document sources of their information, date of information production, and review date of online information updates.

The visual characteristics of a website, including its design and graphic content, attract users and encourage them to spend more time on the website and revisit it (30). The present findings showed that one of the strengths of English SA-related websites was the high quality of their graphic content and maps. In line with our research, Hung and Stones compared children's eHealth design between Eastern and Western countries and reported the superiority of Western websites in terms of information design, multimedia design, and interface design; however, users' needs were better represented in Eastern websites than in Western ones (31).

The present study had some limitations. First, it was preferable to use keywords selected by SA help-seeking patients for searching related websites, as they were not familiar with the medical terminology. Second, it was not possible to ask users about their satisfaction with the websites, since Iranian patients with SA tended to hide their problems due to fear of punishment and feeling of shame. This led to their unwillingness to participate in such studies. On the other hand, our study may be helpful as it presents a list of websites that provide the highest quality of information on SA. However, it should be noted that the Internet is continuously evolving and that the quality of websites may change over time or new high-quality websites may be developed. In future, it is recommended to determine whether culture can affect the quality of online websites and how it influences the quality assessment of these websites.

\subsection{Conclusions}

Based on evidence-based medicine, it is important to extend health-related websites. Moreover, it is essential to notify internet users about the quality of the information content and structure of health-related websites according to specialized quality measurement. In particular, in taboo topics such as sex, Iran as a closed religious society is not able to present sexual education, information, and guidance to its people. Anonymity while web browsing is an opportunity for Iranian people to search for their sexual problems and have freedom to express their requests and needs without embarrassment.

\section{Acknowledgments}

The authors would like to thank medical students for their collaboration.

\section{Footnotes}

Authors' Contribution: Raheleh Rafaiee designed the study and wrote the draft of the manuscript. Pooria Sobhanian Kafshgarkolaee and Parisa Enayati collected the data. Ali Asghar Nadi Ghara analyzed the data. Parisa Enayati was involved in planning, supervised the research, and worked on the manuscript. All the authors discussed the results and commented on the manuscript.

Conflict of Interests: The authors do not have any conflicts of interest to disclose.

Funding/Support: No funding to declare.

\section{References}

1. Wang L, Wang J, Wang M, Li Y, Liang Y, Xu D. Using Internet search engines to obtain medical information: A comparative study. J Med Internet Res. 2012;14(3). e74. doi: 10.2196/jmir.1943. [PubMed: 22672889]. [PubMed Central: PMC3799567].

2. Powell JA, Darvell M, Gray JA. The doctor, the patient and the world-wide web: How the internet is changing healthcare. $J R S o c$ Med. 2003;96(2):74-6. doi: 10.1258/jrsm.96.2.74. [PubMed: 12562977]. [PubMed Central: PMC539397].

3. Siron S, Dagenais C, Ridde V. What research tells us about knowledge transfer strategies to improve public health in low-income countries: A scoping review. Int J Public Health. 2015;60(7):849-63. doi: 10.1007/s00038-015-0716-5. [PubMed: 26298445]. [PubMed Central: PMC4636521].

4. Khalesi ZB, Simbar M, Azin SA. A qualitative study of sexual health education among Iranian engaged couples. Afr Health Sci. 2017;17(2):38290. doi: 10.4314/ahs.v17i2.12. [PubMed: 29062333]. [PubMed Central: PMC5637023]. 
5. Kraus SW, Voon V, Potenza MN. Should compulsive sexual behavior be considered an addiction? Addiction. 2016;111(12):2097106. doi: 10.1111/add.13297. [PubMed: 26893127]. [PubMed Central: PMC4990495].

6. Kuzma JM, Black DW. Epidemiology, prevalence, and natural history of compulsive sexual behavior. Psychiatr Clin North Am. 2008;31(4):603-11. doi:10.1016/j.psc.2008.06.005.[PubMed: 18996301].

7. Yasan A, Essizoglu A, Yildirim EA. Predictor factors associated with premarital sexual behaviors among university students in an Islamic culture. Int J Sex Health. 2009;21(3):145-52. doi: 10.1080/19317610903113813.

8. Javadnoori M, Roudsari RL, Hasanpour M, Hazavehei SM, Taghipour A. Female adolescents' experiences and perceptions regarding sexual health education in Iranian schools: A qualitative content analysis. Iran J Nurs Midwifery Res. 2012;17(7):539-46. [PubMed: 23922603]. [PubMed Central: PMC3730460].

9. Moshtagh M, Rafiey H, Mirlashari J, Azin A, Farnam R. Facilitators of and barriers to compulsive sexual behavior in Iranian women. Sex Addict Compulsivity. 2017;24(4):270-84. doi: 10.1080/10720162.2017.1358124.

10. Fong TW. Understanding and managing compulsive sexual behaviors. Psychiatry (Edgmont). 2006;3(11):51-8. [PubMed: 20877518]. [PubMed Central: PMC2945841].

11. Lewczuk K, Szmyd J, Skorko M, Gola M. Treatment seeking for problematic pornography use among women. J Behav Addict. 2017;6(4):445-56. doi: 10.1556/2006.6.2017.063. [PubMed: 29034717]. [PubMed Central: PMC6034965].

12. Kauer SD, Mangan C, Sanci L. Do online mental health services improve help-seeking for young people? A systematic review. J Med Internet Res. 2014;16(3). e66. doi: 10.2196/jmir.3103. [PubMed: 24594922]. [PubMed Central: PMC3961801].

13. Latifnejad Roudsari R, Javadnoori M, Hasanpour M, Hazavehei SM, Taghipour A. Socio-cultural challenges to sexual health education for female adolescents in Iran. Iran J Reprod Med. 2013;11(2):101-10. [PubMed: 24639734]. [PubMed Central: PMC3941358].

14. Fiksdal AS, Kumbamu A, Jadhav AS, Cocos C, Nelsen LA, Pathak J, et al. Evaluating the process of online health information searching: $A$ qualitative approach to exploring consumer perspectives.J Med Internet Res. 2014;16(10). e224. doi: 10.2196/jmir.3341. [PubMed: 25348028]. [PubMed Central: PMC4285658].

15. Cameron KA, Salazar LF, Bernhardt JM, Burgess-Whitman N, Wingood GM, DiClemente RJ. Adolescents' experience with sex on the web: Results from online focus groups. J Adolesc. 2005;28(4):535-40. doi: 10.1016/j.adolescence.2004.10.006. [PubMed:16022887].

16. Kuss DJ, Griffiths MD, Karila L, Billieux J. Internet addiction: A systematic review of epidemiological research for the last decade. Curr Pharm Des. 2014;20(25):4026-52. doi: 10.2174/13816128113199990617. [PubMed: 24001297].

17. Cooper AL, Delmonico DL, Griffin-Shelley E, Mathy RM. Online sexual activity:An examination of potentially problematic behaviors. Sex Addict Compulsivity. 2004;11(3):129-43. doi:10.1080/10720160490882642.

18. van Lankveld JJ, Leusink P, van Diest S, Gijs L, Slob AK. Internet-based brief sex therapy for heterosexual men with sexual dysfunctions: A randomized controlled pilot trial. J Sex Med. 2009;6(8):2224-36. doi: 10.1111/j.1743-6109.2009.01321.x. [PubMed: 19493295].
19. Gill KM, Hough S. Sexuality training, education and therapy in the healthcare environment: Taboo, avoidance, discomfort or ignorance? Sex Disabil. 2007;25(2):73-6. doi: 10.1007/s11195-007-9033-0.

20. Van Diest SL, Van Lankveld JJ, Leusink PM, Slob AK, Gijs L. Sex therapy through the internet for men with sexual dysfunctions: A pilot study. J Sex Marital Ther. 2007;33(2):115-33. doi: 10.1080/00926230601098456. [PubMed: 17365513].

21. Sevcíkova A, Blinka L, Soukalova V. Excessive internet use for sexual purposes among members of sexaholics anonymous and sex addicts anonymous. Sex Addict Compulsivity. 2018;25(1):65-79. doi: 10.1080/10720162.2018.1431166.

22. Mohseni F, Rafaiee R. Results of activity of anonymous alcoholic association in Iran. Addict Health. 2018;10(1):11-6. doi: 10.22122/ahj.v10i1.554. [PubMed: 30627380]. [PubMed Central: PMC6312557]

23. Wells B. Self-help groups. The international handbook of addiction behaviour. 2016. p. 254-7.

24. von Ranson KM, Farstad SM. Self-help approaches in the treatment of eating disorders, substance use disorders, and addictions. Eating disorders, addictions and substance use disorders. Springer; 2014. p. 587608. doi: 10.1007/978-3-642-45378-6_27.

25. The growing trend of rape in Iran. 2017. Available from: https://fa shafaqna.com/news/414860/.

26. Sex addicted. 2018. Available from: http://sa-iran.org/.

27. Eysenbach G, Powell J, Kuss O, Sa ER. Empirical studies assessing the quality of health information for consumers on the world wide web: A systematic review. JAMA. 2002;287(20):2691-700. doi: 10.1001/jama.287.20.2691. [PubMed: 12020305].

28. McInerney C, Bird N. Assessing Website quality in context: Retrieving information about genetically modified food on the Web. Inform Res. 2004;10(2):213.

29. Harland J, Bath P. Assessing the quality of websites providing information on multiple sclerosis: Evaluating tools and comparing sites. Health Informatics J. 2007;13(3):207-21. doi: 10.1177/1460458207079837. [PubMed: 17711882].

30. Hasan L, Abuelrub E. Assessing the quality of web sites. Appl Comput Informat. 2011;9(1):11-29. doi:10.1016/j.aci.2009.03.001.

31. Hung YL, Stones C. A comparative study of children's eHealth design between East and West: A case study of children's health Websites in China, Taiwan, the UK, and the US. International Conference on Ergonomics and Health Aspects of Work with Computers. Berlin, Heidelberg: Springer; 2011. p. 129-38.

32. Zhang Y, Sun Y, Xie B. Quality of health information for consumers on the web: A systematic review of indicators, criteria, tools, and evaluation results. J Assoc Inform Sci Tech. 2015;66(10):2071-84. doi 10.1002/asi.23311.

33. Raj S, Sharma VL, Singh AJ, Goel S. Evaluation of quality and readability of health information websites identified through India's major search engines. Adv Prev Med. 2016;2016:4815285 doi: 10.1155/2016/4815285. [PubMed: 27119025]. [PubMed Central: PMC4826908].

34. Croft DR, Peterson MW. An evaluation of the quality and contents of asthma education on the World Wide Web. Chest. 2002;121(4):1301-7. doi: 10.1378/chest.121.4.1301. [PubMed: 11948066]. 